Check for updates

Cite this: Phys. Chem. Chem. Phys., 2020, 22, 21630

Received 3rd June 2020 , Accepted 13th August 2020 DOI: $10.1039 / \mathrm{d} 0 \mathrm{cp} 02994 \mathrm{~g}$ rsc.li/pccp

\title{
Reorganization energies and spectral densities for \\ electron transfer problems in charge transport materials $\dagger$
}

\begin{abstract}
Chao-Ping Hsu (iD)
In describing the dynamics of electron transfer or charge transport, the reorganization energy and the spectral density function describe the influence of nuclei motion to the transporting electron. The spectral density can be obtained using various theoretical approaches: from a model of dielectric response, or calculated with various computational means. With the vast advancement of modern computational techniques, many details in vibronic coupling can be obtained, including those described in the early literature. In this work, we provide a comprehensive understanding of the nature of vibronic coupling in light of some early literature. The theoretical connection among different quantities for vibronic coupling is discussed, followed by a brief review of the spectral density function. Various approaches and some of the results for the spectral density function are also reviewed. The importance of low-frequency bands in nonpolar systems that can be overlooked is also discussed, for both Holstein and Peierls types of electron-phonon couplings.
\end{abstract}

128 Academia Road Section 2, Institute of Chemistry, Academia Sinica, Nankang, Taipei, 11529, Taiwan. E-mail: cherri@sinica.edu.tw; Fax: +886 2 27831237; Tel: +886255728658

$\dagger$ This article is dedicated to the memory of Prof. Ching-Liang Lin (1931-2019), for her warm love to students and her firm support to women physicists.

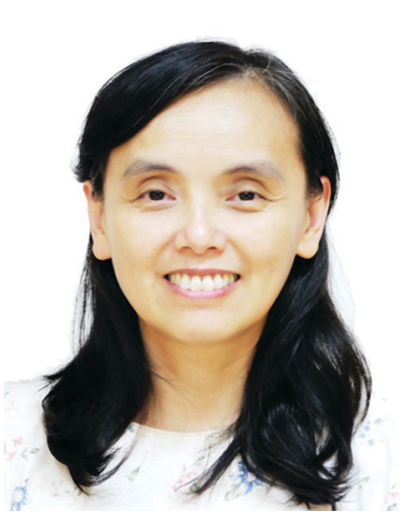

Chao-Ping Hsu
Chao-Ping (Cherri) Hsu is a research fellow at the Institute of Chemistry, Academia Sinica located in Taiwan. She developed computational schemes for electronic coupling in electron transfer, energy transfer and singlet fission with firstprinciples quantum chemistry computation. She is also interested in developing mathematical models for understanding noises and dynamics systems biology. Major awards and recognitions she has received include the Pople Medal

from the Asia-Pacific Association of Theoretical \& Computational Chemists in 2010, the Outstanding Research Award from the National Science Council in 2011, and the Investigator Award from Academia Sinica 2017.

\section{Introduction}

The theory of electron transfer (ET) was developed more than half a century ago. ${ }^{1,2}$ The rich insights provided, and the great potential in many applications, have triggered enormous progress in many different fields. One area that brought great impact to our society has been the development of charge transport materials, especially in organic molecular devices. Organic semiconductors are important for many optoelectronic applications, ${ }^{3-5}$ including in field-effect transistors, ${ }^{6}$ light-emitting diodes, ${ }^{7}$ photovoltaic cells, ${ }^{8}$ etc. As compared with their silicon-based counterparts, organic semiconductors are advantageous for their low power consumption, low cost and compatibility for large-area fabrication. ${ }^{9}$ Organic semiconductors are also important for solar energy conversion, in that hole- and electron-transporting layers are now commonly used in perovskite solar cells. ${ }^{10}$ In these applications, charge mobility has been one of the key properties of semiconducting devices. ${ }^{11}$ Theoretically predicting the charge mobility is highly desirable for physical insights and the chance to further improve the design of new materials.

To develop systems with good charge transport, non-polar (or less dipolar) molecules with low band gaps have been commonly considered, for the benefit of having low reorganization energy (often denoted as $\lambda$ ). One of the key insights of Marcus theory is that the activation energy of the reaction arises essentially from the reorganization of polarization from the environment. The less polar molecular device offers an environment with low dielectric response, which is quite different from systems 
discussed in the traditional electron transfer (ET) literature, where electrolytes are mainly in a polar or even aqueous solution. Most of the insights derived previously with polar environments in mind might still be valid in the nonpolar systems. Therefore, one of the goals of the present work is to lay out aspects of the reorganization energy with a stress on our current understanding of a nonpolar system.

With the vast advancement of computational hardware and software, it is now routinely possible to calculate and dissect the reorganization energy in various forms. Reorganization energy, electron-phonon coupling, and spectral density function are frequently discussed in the ET literature or related topics. Modern works simulating the charge transport dynamics have been developed using various schemes. ${ }^{12-18}$ However, because of the complex nature of the problem, there are inevitable discrepancies among different theoretical models and simulation approaches. For example, to date, most charge transport simulation works rarely considered the feedback of the polaron to the classical nuclear motions, whereas recent improvements have been developed ${ }^{19-21}$ to include such feedbacks. Some early literature, mainly in the field of non-equilibrium statistical mechanics, has offered rigorous and insightful bases, such that modern computational work can be relied on, helping us derive further understanding for the system. $^{22-26}$ Many of these works are well-written, with great insights; some do not have apparent relevance, whereas others might appear to be difficult to understand especially in the theoretical derivations. For facilitating the development of modern simulation, a summary and overview on the theoretical grounds is helpful, which is what we aim to provide in the present work. Therefore, another goal of the present work was to introduce such works and connect their results to the modern computational chemistry. Therefore, this work is far from any comprehensive review for modern computational chemistry work on charge-transport materials. Instead, we aim to provide an integrated and yet modest set of relevant information from the traditional statistical mechanics field, for deriving more insights from computational results.

In this work, we first review various similar quantities for the reorganization energy and their background theoretical models, followed by a section on the dielectric continuum models and their theoretical predictions. We then outline some of the computational chemistry results and conclude with speculation on future perspectives.

\section{Reorganization energy and its similar quantities in various models}

For describing the process of charge transfer, the Marcus ET theory proposed a rate expression, ${ }^{1,2}$ which is determined by 3 parameters: the Gibbs standard free energy for the reaction $\Delta G^{0}$, the electronic coupling $H_{\mathrm{RP}}$, and the reorganization energy $\lambda$ :

$$
k_{\mathrm{ET}}=\frac{2 \pi}{\hbar} \frac{\left|H_{\mathrm{RP}}\right|^{2}}{\sqrt{4 \pi \lambda k_{\mathrm{B}} T}} \exp \left(-\frac{\left(\Delta G^{0}+\lambda\right)^{2}}{4 \lambda k_{\mathrm{B}} T}\right),
$$

where $k_{\mathrm{B}}$ is the Boltzmann constant. One of the most important insights for the Marcus ET rate theory is in the treatment of the solvent and its influence. Because ET involves a change in the location of a charge, it drives changes in many degrees of freedom of a presumably polar environment, and these changes in the environment feed back to the system. Such changes in the environment collectively form the reaction coordinate. The free energy of the reactant and product state in this reaction coordinate was assumed to be a parabola, an assumption that was later confirmed to hold until achieving very high energy. ${ }^{27}$ We outline several different charge transfer/transport models below, with a stress on their definitions and treatments of the quantities that are similar to the reorganization energy.

\subsection{Reorganization energy in the Marcus theory}

The reorganization energy is a critical quantity in ET theory. It is the energy required to reach the relaxed product nuclear configurations from a relaxed reactant configuration, while keeping the electronic state in the reactant (or vice versa). If the difference in equilibrium positions of the two parabolas is defined as 1 (arbitrary unit in the reaction coordinates), then one potential energy curve can be $\lambda R^{2}$, and the other is $\lambda(R-1)^{2}+\Delta G^{0}$, with $R$ being the position in the generalized reaction coordinate. Therefore, $\lambda$ can also be considered as the curvature for the Marcus parabola (Fig. 1(a)).

We note that, for a classical harmonic oscillator, a standard theoretical model does not need any parameter, because the units for displacement and the energy can be chosen to fit any harmonic oscillator in application. For ET problems, the minimal number of parameters describing the set of two parabola with the same curvature is 2 , with one being the free-energy difference $\Delta G^{0}$, measuring the vertical shift of the two curves, and another can be defined by various means: the curvature, the displacement (horizontal shift), or the coefficient for the difference of the two states (which is a linear function), depending on the setting of the theoretical models.

The reorganization energy is further divided into two different contributions: one arising from the surrounding solvent, the outer sphere component, $\left(\lambda_{\text {out }}\right)$ and the other from internal degrees of freedom (or the first solvation shell, $\lambda_{\text {in }}$ ).

For $\lambda_{\text {out }}$, because the process of ET involves a change in charge distribution of the donor-acceptor pair, the most important effect from the environment is its polarization, or the dielectric response. An expression considering such changes, in a simplified spherical cavity model, was given by Marcus: ${ }^{28,29}$

$$
\lambda_{\text {out }}=(\Delta e)^{2}\left(\frac{1}{2 r_{\mathrm{D}}}+\frac{1}{2 r_{\mathrm{A}}}-\frac{1}{R_{\mathrm{DA}}}\right)\left(\frac{1}{\varepsilon_{\mathrm{op}}}-\frac{1}{\varepsilon_{\mathrm{S}}}\right),
$$

where $\Delta e$ is the amount of charge transferred in the reaction; $r_{\mathrm{D}(\mathrm{A})}$ is the radius of the donor (acceptor), when a spherical cavity is used to model the molecule or fragment; $R_{\mathrm{DA}}$ is the center-tocenter distance between the donor and the acceptor; $\varepsilon_{\mathrm{op}}$ is the optical dielectric constant; and $\varepsilon_{\mathrm{S}}$ is the static dielectric constant for the solvent. The expression in eqn (2) was derived from a model where the dielectric solvation energy change was estimated by two independent spheres (infinitely separated) containing the donor and the acceptor, and the changes in the interaction energy between the two (the term involving $1 / R_{\mathrm{DA}}$ ). The optical dielectric 
(a)

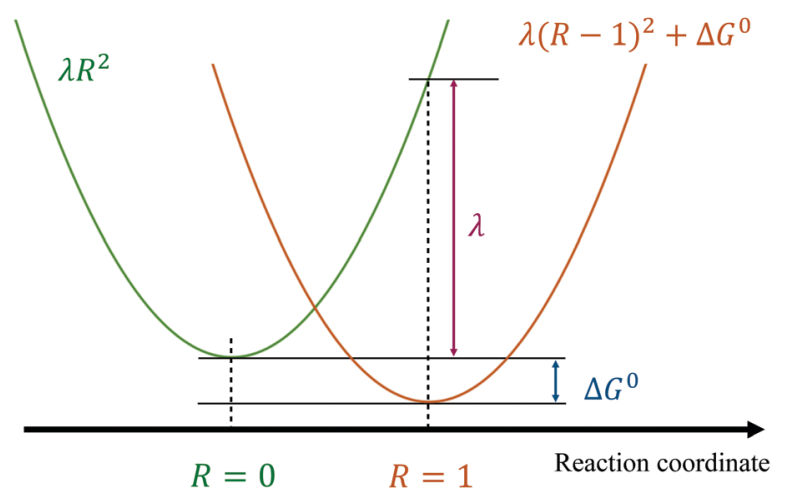

(b)

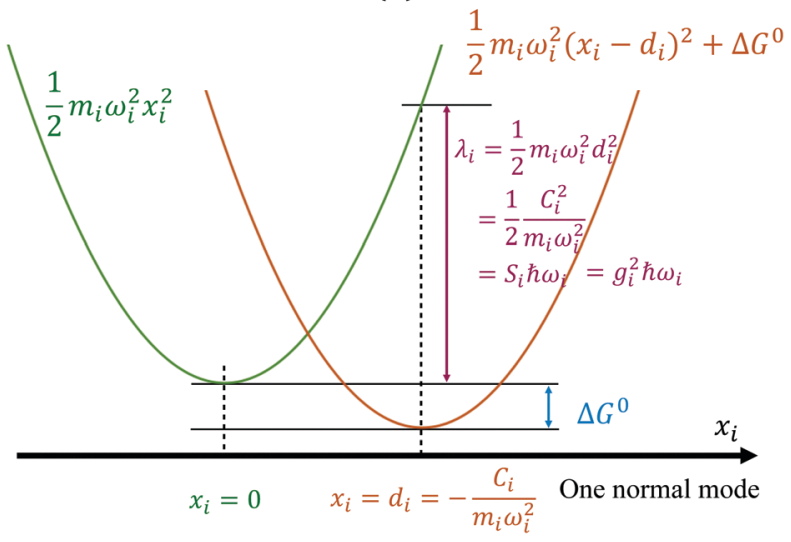

Fig. 1 Marcus' parabolic free energy curves (a), broken down into harmonic oscillators, showing one of them as $x_{i}$ as shown in (b), with various quantities labelled.

response follows the electron being transferred instantaneously, and this part does not contribute to the reorganization energy. In practice, $\lambda_{\text {out }}$ can be quite sensitive to the parameters $r_{\mathrm{D}(\mathrm{A})}$ and $R_{\mathrm{DA}}$ chosen. $r_{\mathrm{D}(\mathrm{A})}$ can be estimated from the sphere that has the same van der Waals volume of the donor or acceptor fragments, and $R_{\mathrm{DA}}$ is the distance between the centers of mass of the fragments.

Another component for reorganization is the internal contribution, which is derived from the internal degrees of freedom for a molecule, or the inner solvation shell for an ion in a polar solution. In this case it is common to express the reorganization energy in terms of the structural difference $(\Delta \vec{q})$ in the reactant and product states and project such a structural difference to the normal modes (in mass-weighted coordinates $\left\{Q_{i}\right\}$ ) of the reactant (or the product).

$$
\lambda_{\text {in }}=\sum_{i} \frac{1}{2} m_{i} \omega_{i}^{2} d_{i}^{2}=\sum_{i} \lambda_{i},
$$

with $d_{i}$ being the projection of the structural difference, the displacement, to the $i$-th normal mode,

$$
\sqrt{m_{i}} d_{i}=\Delta \vec{q} \cdot \hat{Q}_{i}
$$

It is common to use the dimensionless Huang-Ruy factor $\left\{S_{i}\right\}$ to describe the contribution of each mode,

$$
\lambda_{i}=S_{i} \hbar \omega_{i},
$$

with $S_{i}=m_{i} \omega_{i} d_{i}^{2} / 2 \hbar$ (as summarized in Fig. 1(b)). Because the vibrational quanta can be closer to or higher than the thermal energy $k_{\mathrm{B}} T$, such a breakdown is convenient for including quantum statistics. ${ }^{23}$

\subsection{Spin-boson Hamiltonian}

The Spin-Boson Hamiltonian (SBH) has been used to study a broad range of dissipative problems. ${ }^{30}$ In $\mathrm{SBH}$, a set of harmonic oscillators are included to model the environment, affecting the dynamics of a simple two-state system.

$$
\hat{H}=\frac{\varepsilon}{2} \sigma_{z}+\frac{V}{2} \sigma_{x}+H_{\mathrm{B}}+H_{\mathrm{SB}},
$$

where the Pauli spin matrices $\sigma_{x}$ and $\sigma_{z}$ are used to denote the $2 \times 2$ Hamiltonian, with $|+\rangle$ and $|-\rangle$ as their basis. In this case, $\sigma_{x}=|+\rangle\langle-|+|-\rangle\langle+|$ and $\sigma_{z}=|+\rangle\langle+|-|-\rangle\langle-| . H_{\mathrm{B}}$ is the Hamiltonian for the environment (bath), and $H_{\mathrm{SB}}$ is the interaction of the system and bath,

$$
\begin{gathered}
H_{\mathrm{B}}=\sum_{i}\left(\frac{p_{i}}{2 m_{i}}+\frac{1}{2} m_{i} \omega_{i}^{2} x_{i}^{2}\right), \\
H_{\mathrm{SB}}=\frac{1}{2} \sigma_{z} \sum_{i} C_{i} x_{i} .
\end{gathered}
$$

The ladder operators in the second quantization of harmonic oscillator, $b_{i}^{\dagger}$ and $b_{i}$ can also be used in the expression:

$$
\begin{gathered}
H_{\mathrm{B}}=\hbar \omega_{i}\left(b_{i}^{\dagger} b_{i}+\frac{1}{2}\right), \\
H_{\mathrm{SB}}=\frac{1}{2} \sigma_{z} \sum_{i} C_{i}^{\prime}\left(b_{i}^{\dagger}+b_{i}\right),
\end{gathered}
$$

where

$$
C_{i}^{\prime}=C_{i} \sqrt{\frac{\hbar}{2 m_{i} \omega_{i}}} .
$$

Namely, $C_{i}$ is the coefficient of the linear vibonic coupling in the classical mechanical expression, and $C_{i}^{\prime}$ is essentially the same quantity but with dimensions adjusted for the second quantization expression. With $C_{i} x_{i}$ being the coupling from mode $i$, the displacement of the two states is

$$
d_{i}=-\frac{C_{i}}{m_{i} \omega_{i}^{2}},
$$

leading to a contribution to the reorganization energy

$$
\lambda=\sum_{i} \frac{1}{2} \frac{C_{i}^{2}}{m_{i} \omega_{i}^{2}} .
$$

Fig. 1(b) summarizes the quantities in the parabola for each mode $i$. The two-state SBH is similar to the many-state Holstein models developed for describing polaron transport, as outlined below. Breaking down to Harmonic oscillators is intuitively 
straightforward for $\lambda_{\text {in }}$, because it arises from displaced vibrational modes of the donor and the acceptor. However, for SBH (and also the Holstein and/or Peierls models), such a break-down can be generalized to all the external influences to the states, both inner and outer-sphere contributions.

\subsection{Holstein and Peierls models}

For an array of organic semiconducting molecules, it is common to consider a Hamiltonian ${ }^{31-35}$ as

$$
H=\sum_{n m} t_{n m} a_{n}^{\dagger} a_{m}+\sum_{n} \varepsilon_{n} a_{n}^{\dagger} a_{n}+\sum_{n, i} \hbar \omega_{i}\left(b_{n, i}^{\dagger} b_{n, i}+\frac{1}{2}\right)+H_{\mathrm{ep}},
$$

which contains electronic interaction between sites $t_{m n}$, electronic site energy $\varepsilon_{n}$, the Hamiltonian for vibrations or phonons, and the electron-phonon interaction $H_{\text {ep }}$, respectively. In eqn (14), $a_{n}^{\dagger}$ and $a_{n}$ are the electronic creation and annihilation operators at site $n$, and $\varepsilon_{n}$ is the site energy, $b_{n, i}^{\dagger}$ and $b_{n, i}$ are the creation and annihilation operators for the vibration associated with site $n$ and the $i$ th mode, and $\omega_{i}$ denotes vibration frequencies. It is common to include the local coupling, or the Holstein's Hamiltonian, in the following form,

$$
H_{\mathrm{ep}}^{\mathrm{H}}=\sum_{n, i} g_{i}^{\mathrm{H}} \hbar \omega_{i} a_{n}^{\dagger} a_{n}\left(b_{n, i}^{\dagger}+b_{n, i}\right),
$$

$g_{i} \omega_{i}$ is the electron-phonon coupling, and where the $n$th electronic basis is essentially the site (position) of the charge, and its energy is coupled to vibrational mode $i$ at site $n .^{33}$ Typically, in modeling organic semiconductors, each organic molecule constitutes a site, with vibrational modes $i$ being assigned to each site. Here it is assumed that the vibrational modes are localized to one or a finite cluster of molecules, such that frequency $\omega_{i}$ can be used for site $n$. In a periodic crystal system, it is common to describe the system in the Fourier transformed bases, turning vibration into phonons of wave vector $q$,

$$
H_{\mathrm{ep}}^{\mathrm{H}}=\sum_{n, i, q} g_{i q}^{\mathrm{H}} \hbar \omega_{i q} a_{n}^{\dagger} a_{n}\left(b_{i,-q}^{\dagger}+b_{i, q}\right) .
$$

with $b_{i q}$ being the annihilation operator for the phonon mode $i$ with wavevector $q$.

It is also possible to further generalize the electron-phonon coupling to the transfer integral, leading to the Peierl electronphonon coupling, which is also studied in the literature ${ }^{36-38}$

$$
H_{\mathrm{ep}}^{\mathrm{P}}=\sum_{m \neq n, i, q} g_{i q, m n}^{\mathrm{P}} \hbar \omega_{i q} a_{m}^{\dagger} a_{n}\left(b_{i,-q}^{\dagger}+b_{i, q}\right) \text {. }
$$

In both electron-phonon coupling models, $g_{i}$ is a dimensionless parameter for electron-phonon coupling. It can be shown that

$$
g_{i} \hbar \omega_{i}=C_{i}^{\prime}=C_{i} \sqrt{\frac{\hbar}{2 m_{i} \omega_{i}}},
$$

where coefficients $C$ and $C^{\prime}$ share the same definition for the SBH (eqn (11)). The contribution to the reorganization energy is, with eqn (13)

$$
\lambda_{i}=\frac{C_{i}^{2}}{2 m_{i} \omega_{i}^{2}}=\frac{C_{i}^{\prime 2}}{\omega_{i} \hbar}=g_{i}^{2} \hbar \omega_{i}
$$

We note that all the parameters for electron-phonon coupling, $g$ and $C\left(C^{\prime}\right)$ have their counterparts in the off-diagonal Peierls coupling.

\subsection{On the locality of electron-phonon coupling}

In the literature, the term "local" electron-phonon coupling often refers to the Holstein model, whereas "nonlocal" indicates the Peierl electron-phonon coupling. ${ }^{39,40}$ For this purpose, the terms "diagonal" and "off-diagonal" coupling might be more intuitive. ${ }^{41,42}$ With an array of molecules in mind, the idea of "local" coupling can also refer to the physical location of the electron and vibration, assuming that they are described in a local basis. Because it is natural to develop physical pictures with real space and molecules in mind, here we discuss the idea of "locality" in this aspect. For example, a "non-local" Holstein model can be defined as:

$$
H_{\mathrm{ep}}^{\mathrm{H}}=\sum_{n, i} g_{i, n-m}^{\mathrm{H}} \hbar \omega_{i} a_{n}^{\dagger} a_{n}\left(b_{m, i}^{\dagger}+b_{m, i}\right),
$$

where a movement or vibration at site $m$ could affect the energy at site $n$, and thus $g$ carries an index for mode $i$, as well as the relative position in the site indexes $m$ and $n$. Because a nuclear movement (vibration) at a certain location can affect a limited range of electrons nearby, both in the diagonal or off-diagonal Hamiltonian, the coupling factor $g$ has some local nature in the real space. A very local, single-site coupling (eqn (15)) leads to a "universal" coupling in the reciprocal space, where $g_{i q}^{\mathrm{H}}$ is a constant of $q \cdot{ }^{43}$ Such a simplified setting certainly allows for much easier theoretical manipulation. In practice, because electrostatic interaction that dominates the site energy fluctuation is long range in nature, the coupling should be range-dependent.

However, we note that, for an array of molecules, periodic or not, assigning a single-site location for a vibrational mode in general can be difficult. As eigenvectors of the Hessian matrix, the motion of nuclei can be distributed all over the system. Expressions such as eqn (15) and (20) were written when the coupling among local vibrations is not large, or, in an amorphous condensed phase where vibrational modes are somewhat localized to certain sites. Therefore, the frequently used expression in eqn (16) is more general and could better describe the long-range interaction between a general vibrational mode $q$ and site $n$.

A great proportion of interesting problems in chemistry are not in the gas phase, nor do they take place in a periodic environment. The classical redox systems mainly involve electrolytes in solutions, and the modern charge transporting devices are mostly in amorphous solid-state phase, not to mention that many interesting ET problems are in biomolecules such as proteins or DNAs, mainly in aqueous solution. A convenient treatment for the model is to keep electrons in a desirable basis, $\neq$ and to use vibrational modes for the system as a whole. Depending on the computational setting, usually it is easy to separate the inner-shell contribution, the vibrational modes of the donor and acceptor molecules (or fragments) from those arising from the surrounding

$\ddagger$ The electrons can be described with adiabatic ${ }^{13,14,16,21}$ or diabatic basis, ${ }^{44}$ but the discussion is out of the scope of the present work. 
molecules. Here we note that the expression in eqn (17) can be generalized further, with an index for all vibrational modes of the whole system, collective or not. ${ }^{38,45}$ The Holstein-Peierls (HP) coupling term can be expressed as,

$$
H_{\mathrm{ep}}^{\mathrm{HP}}=\sum_{m, n, i} g_{i, m n}^{\mathrm{P}} \hbar \omega_{i} a_{m}^{\dagger} a_{n}\left(b_{i}^{\dagger}+b_{i}\right),
$$

which allows both Holstein $(m=n)$ and Peierls $(m \neq n)$ types of coupling, regardless of the location of electrons and phonons/ vibrations.

\subsection{Spectral density function}

For both the SBH and HP models, one commonly defines a spectral density function $J(\omega)$ to represent the coupling strength of modes of frequency $\omega$. It can be defined with $C_{i}$, the strength of coupling for mode $i$, as,

$$
J(\omega)=\frac{\pi}{2} \sum_{i} \frac{C_{i}^{2}}{m_{i} \omega_{i}} \delta\left(\omega-\omega_{i}\right) .
$$

The propagation of the two-level dynamics requires a treatment for the vibronic coupling $\sigma_{z} \sum_{i} C_{i} x_{i}$, which couples the nuclear and electronic degrees of freedom. This is often achieved by the a time-dependent perturbation theory under the interaction picture of quantum mechanics. Such techniques have been successful in treating many problems in quantum dynamics, ${ }^{46,47}$ including nonlinear spectroscopy ${ }^{48}$ and the two-state reaction rates with or beyond Fermi's golden rule. ${ }^{49}$ In these works, a central quantity $X$ is defined as the energy difference between the two states in $\mathrm{SBH}$, or the change in energy when the site carries a charge for the Holstein model,

$$
X=\sum_{i} C_{i} x_{i}
$$

and the time evolution of this operator in the ensemble of one of the states is central in describing the dissipative two-state dynamics.

$$
X(t)=\mathrm{e}^{i t H / \hbar} X \mathrm{e}^{-i t H / \hbar},
$$

where $H$ is the Hamiltonian of the system. Typically the offdiagonal part of $H$ is separated and treated with perturbation expansion. ${ }^{46,47}$ The time-correlation function for $X(t)$ can be calculated and related to the spectral density function. With the correlation function of harmonic oscillators, we have,

$$
\begin{gathered}
C(t) \equiv\langle\delta X(t) \delta X(0)\rangle \\
=\frac{\hbar}{\pi} \int_{0}^{\infty} \mathrm{d} \omega J(\omega)[\operatorname{coth}(\beta \hbar \omega / 2) \cos \omega t-i \sin \omega t],
\end{gathered}
$$

with $\beta=1 / k_{\mathrm{B}} T$. With the fluctuation-dissipation theorem, $C(t)$ is also related to the response of the system when an external change takes place.

With $C_{i} x_{i}$, the displacement of each mode is $C_{i} / m_{i} \omega_{i}{ }^{2}$, and the contribution to the reorganization energy is $C_{i}^{2} / 2 m_{i} \omega_{i}{ }^{2}$. Therefore, the reorganization can be related to $J(\omega)$ as well:

$$
\lambda=\frac{1}{\pi} \int_{0}^{\infty} \frac{J(\omega)}{\omega} \mathrm{d} \omega .
$$

Just as $\lambda$ is divided into the inner and outer-shell contribution, the spectral density can also be divided in the same way. The inner-shell component is much easier to define. As discussed in Section 2.1, the individual contribution $\lambda_{i}$ can be defined and calculated routinely (eqn (3)). Therefore the "inner" spectral density function can be

$$
J_{\text {in }}(\omega)=\pi \sum_{i} \lambda_{i} \omega_{i} \delta\left(\omega-\omega_{i}\right) .
$$

Thus, in principle, we should have $J(\omega)=J_{\text {in }}(\omega)+J_{\text {out }}(\omega)$. However, because $J_{\text {out }}(\omega)$ is much less trivial to obtain and will be the main focus in the present work, for the sake of simplicity and to follow the convention of the major literature, we will use $J(\omega)$ for $J_{\text {out }}(\omega)$ when it is not confusing.

In theoretical studies, one commonly uses analytical functions such as

$$
J(\omega)=A \omega^{s} \mathrm{e}^{-\omega / \omega_{\mathrm{c}}},
$$

with $s=1$ being the ohmic dissipation. Models for $s>1$ (superohmic) and $s<1$ (sub-ohmic) are also discussed. ${ }^{30}$ Such expressions are convenient. With only 3 parameters, there is a good chance to take $J(\omega)$ fully into account in analytical theories. However, these Ohmic and similar models are more-or-less phenomenological, since there are a lot of important details in $J(\omega)$ that are not included, as outlined below. Realistic models can be developed for calculating $J(\omega)$ with known physical properties of the system (Section 3), or simulating it (Section 4). Such approaches eliminate the need to fit the parameters and offer chances to predict and design new systems with desirable properties.

We note that in Marcus theory, all the influence of the nuclei motion is integrated into $\lambda$. The internal vibrational modes of the donor and acceptor contribute to $\lambda_{\text {in }}$, which comes with a specific set of frequencies. The polarization of the environment constitutes $\lambda_{\text {out }}$, which usually forms a "band" of continuous modes. However, in the SBH and HP models, all the nuclei motions are treated with the harmonic oscillator models, with an infinite number of harmonic oscillators as the model onto which all influences can be projected. ${ }^{25}$ Such theoretical models are flexible for many different problems, but model setting for this infinite number of coupling coefficients becomes a problem. The dielectric continuum models introduced in the following section are practical solutions.

The hypothetical 1-dimensional reaction coordinate is also a central concept in Marcus theory. The harmonic oscillator model for the bath naturally leads to a many (or infinite) dimensional picture, with each dimension similar to that depicted in Fig. 1(b). However, the dynamics of the system can be described using a 1-dimensional model, and the simplest definition for this coordinate is the energy difference between the reactant and product states, $\Delta E(\equiv X)$. As will be shown in the following section and the references cited therein, $\Delta E$ offers a means to model, calculate and understand the dynamics of the system. The fluctuation-dissipation theorem and its corresponding (linear) response function can be built with $\Delta E$. Moreover, a parabolic free energy curve along the reaction coordinate implies a Gaussian distribution in the ensemble. ${ }^{27}$ However, the linear assumption 
could be challenged, and it can be corrected with theoretical and numerical efforts. ${ }^{50}$

\section{Dielectric continuum models}

The mathematical form of infinite harmonic oscillators can also be derived from the linear dielectric response, without having to hypothesize with an infinite number of harmonic vibrations. ${ }^{25}$ With proper dielectric models and boundary conditions, the environmental contribution to $J(\omega)$ can be related to the dielectric response $\varepsilon(\omega)$. Here we lay out the theoretical ground for such a treatment, with data derived from experiments included, with which we further discuss the physics and requirements for the spectral densities.

\subsection{Theoretical grounds}

Suppose $\Delta \rho(\mathbf{r})$ is the change in charge distribution in the ET, which can be arbitrarily scaled by a time-dependent factor, $\Delta \rho(\mathbf{r}, t)=f(t) \Delta \rho(\mathbf{r})$. The system goes from an initial to final state with $f(t)$ going from 0 to 1 . The frequency-dependent dielectric constant of the media gives rise to an immediate response (modeled by the optical dielectric constant, $\varepsilon_{\text {op }}$ ) and a delayed dielectric solvation after a time-dependent change. The electrostatic interaction between a system and its surrounding dielectric solvent is also time-dependent, $\Delta E_{\text {int }}(t)$. This energy difference is the vibronic coupling $\sum_{i} C_{i} x_{i}$ which is often named $X$ as a major dynamic variable in many related works. ${ }^{25,46,51,52}$

$\Delta E_{\text {int }}(t)$ can be generally written as

$$
\Delta E_{\text {int }}(t)-\left\langle\Delta E_{\text {int }}\right\rangle(\equiv \delta X(t))=\int_{0}^{\infty} \alpha(t) f(t-\tau) \mathrm{d} \tau,
$$

where $\alpha(t)$ is a generalized susceptibility function, and $f(t)$ is an arbitrary time-denpendent perturbation function. With the fluctuation-dissipation theorem, $\alpha(\tau)$ can be related to the time-correlation for $\Delta E_{\text {int }}(t),{ }^{53-55}$

$$
\alpha(\tau)=\langle[\delta X(\tau), \delta X(0)]\rangle=2 \operatorname{Im} C(\tau) / \hbar,
$$

where $C(t)$ is the auto-correlation function for $\Delta E_{\text {int }}(t)$ (or $X(t)$, as defined in eqn (26)), spectral density $J(\omega)$. Therefore, we have,

$$
J(\omega)=-\operatorname{Im} \tilde{\alpha}(\omega) .
$$

$\Delta E_{\text {int }}(t)$ can be formulated using a range of methods, such as the product of the charge (from the solute in the ET system) and the electric potential generated by the polarized solvent, or the displacement field generated by the solute. $\Delta E_{\text {int }}(t)$ has its Fourier transform as follows,

$$
\Delta \tilde{E}_{\text {int }}(\omega)=\int_{-\infty}^{\infty} \Delta E_{\text {int }}(t) \exp (i \omega t) \mathrm{d} t
$$

It can be formulated with dielectric solvation theories: ${ }^{22,25}$

$$
\Delta \tilde{E}_{\mathrm{int}}(\omega)=-\tilde{f}(\omega) \tilde{\alpha}(\omega)
$$

where $\tilde{f}(\omega)=\int_{-\infty}^{\infty} f(t) \exp (i \omega t) \mathrm{d} t$ is a dimensionless function, which can be an arbitrarily chosen perturbation, and $\alpha(\omega)$ is the response function with frequency $\omega$. The specific form of $\alpha(\omega)$ depends on the setting in the electrostatic model. ${ }^{22,25,56}$

1. An early version with the general formulation mainly for a uniform electric (and displacement) field developed in ref. 22, leading to, $\S$

$$
\tilde{\alpha}(\omega) \sim-\int \mathrm{d} \mathbf{r}\left[1-\frac{1}{\varepsilon(\mathbf{r}, \omega)}\right]\left[\mathbf{D}_{i}(\mathbf{r}) \cdot \Delta \mathbf{D}(\mathbf{r})\right],
$$

where $\mathbf{D}(\mathbf{r})$ is the displacement field at location $\mathbf{r}$, which implies that

$$
J(\omega) \propto \operatorname{Im}\left[1-\frac{1}{\varepsilon(\omega)}\right]=-\frac{\varepsilon^{\prime \prime}(\omega)}{|\varepsilon(\omega)|^{2}},
$$

where the dielectric response is written in its real and imaginary parts, as,

$$
\varepsilon(\omega)=\varepsilon^{\prime}(\omega)+\mathrm{i} \varepsilon^{\prime \prime}(\omega) .
$$

We note that this model did not consider the boundary condition for the solute-solvent interface. Most similar models use a cavity or a boundary for different dielectric constants.

2. For the Marcus' sufficiently separated donor and acceptor model, the dielectric image effect can be neglected, such that

$$
\tilde{\alpha}(\omega)=2 \Delta e^{2}\left(\frac{1}{\varepsilon_{\mathrm{op}}}-\frac{1}{\varepsilon(\omega)}\right)\left(\frac{1}{2 r_{\mathrm{D}}}+\frac{1}{2 r_{\mathrm{A}}}-\frac{1}{R_{\mathrm{DA}}}\right),
$$

leading to

$$
J(\omega) \propto \operatorname{Im}\left(\frac{1}{\varepsilon_{\mathrm{op}}}-\frac{1}{\varepsilon(\omega)}\right) .
$$

which is very similar to the results of the model above.

3. Using the Onsager model, the system can also be modeled as a closely spaced donor and acceptor in a spherical cavity of radius $a$ and change in the dipole moment $\Delta \mu$. In this case we can obtain, $\Phi$

$$
\tilde{\alpha}(\omega)=\frac{\Delta \mu^{2}}{a^{3}} \frac{\left(\varepsilon_{\mathrm{c}}+2\right)}{3}\left[\frac{\varepsilon_{\mathrm{op}}-1}{2 \varepsilon_{\mathrm{op}}+\varepsilon_{\mathrm{c}}}-\frac{\varepsilon(\omega)-1}{2 \varepsilon(\omega)+\varepsilon_{\mathrm{c}}}\right],
$$

where $\varepsilon_{\mathrm{c}}$ is the dielectric constant inside the spherical cavity, which has been modeled as 1 because it is in a vacuum, for a cavity that is occupied by the solute (donor and acceptor for ET). Therefore,

$$
J(\omega)=-\frac{\Delta \mu^{2}}{a^{3}} \operatorname{Im} \frac{\left(\varepsilon_{\mathrm{c}}+2\right)}{3} \frac{\varepsilon(\omega)-1}{2 \varepsilon(\omega)+\varepsilon_{\mathrm{c}}},
$$

since $\varepsilon_{\text {op }}$ represents an infinitely fast dielectric response of the system and can be regarded as a real number.

\subsection{Physical origin of dielectric solvation}

In a dielectric material, various factors can contribute to the dielectric effect, roughly from slow to fast in their time scales:

- movement of the molecules (mainly charged particles),

- changes in the orientation of the polar molecule,

$\S \alpha(\omega)$ has a dimension of energy. Here, for the clarity of theories, an expression similar to eqn (12) of ref. 22 was loosely given without a rigorous ground.

T The expression in eqn (40) was slightly different from that described in ref. 25, by a constant factor $\left(\varepsilon_{\mathrm{c}}+2\right)$, and it is closer to those in ref. 51 and 52 . 
- changes in the bond angle or other internal degrees of freedom of the solvent molecules,

- stretching or suppression of polar bonds, and the polarization of electronic distribution,

- polarization of electrons.

For ET, the donor-acceptor system has a change in its charge distribution. The first term, the movement of charge particles does not really occur in the regular solvents composed of neutral molecules. The second item, the orientational effects in polarization, gives rise to Debye dielectric solvation. For ET, the electronic polarization is faster than the charge transfer process; thus an optical dielectric constant $\varepsilon_{\mathrm{op}}$ is used to represent the "infinite frequency" limit in the theories.

3.2.1 Low frequency: Debye dielectric relaxation. For polar solvents, the Debye dielectric relaxation model can be used to describe the dielectric dispersion $\varepsilon(\omega)$, where the response of polarization was assumed to be an exponential decay function, with lifetime $\tau$. $\tau$ can be considered as the orientational lifetime for a fluctuating solvent. This exponential response yields to a dielectric function as,

$$
\varepsilon(\omega)=\varepsilon_{\mathrm{op}}+\frac{\varepsilon_{\mathrm{s}}-\varepsilon_{\mathrm{op}}}{1-i \omega \tau},
$$

where $\tau$ is the characteristic relaxation time. We note that in most of the literature the dielectric response at the high-frequency limit is often denoted as $\varepsilon_{\infty}$, but here we use $\varepsilon_{\mathrm{op}}$ for better coherence with expressions shown above. With this model, it gives

$$
\varepsilon^{\prime}(\omega)=\varepsilon_{\mathrm{op}}+\frac{\varepsilon_{\mathrm{s}}-\varepsilon_{\mathrm{op}}}{1+\omega^{2} \tau^{2}},
$$

and

$$
\varepsilon^{\prime \prime}(\omega)=\frac{\left(\varepsilon_{\mathrm{s}}-\varepsilon_{\mathrm{op}}\right) \omega \tau}{1+\omega^{2} \tau^{2}} .
$$

Therefore, there should be a diffuse peak at $1 / \tau$ for the spectral function $J(\omega)$ for a polar solvent.

3.2.2 Higher frequency: resonances. When a dielectric material is placed under the oscillatory electric field, the oscillations at various levels, as listed above, affect the polarization of the material. If the frequency is low, the motion of the oscillation follows, leading to a cancellation of the electric field applied, and thus dielectric shielding is obtained (in the real part of $\varepsilon(\omega)$ ). When the frequency of the applied field increases, to a point at which the frequency matches the intrinsic frequency of the oscillation, a resonance is observed and there is an absorption of electric energy. An absorption peak (dielectric loss) appears in the imaginary part of $\varepsilon(\omega)$. For frequencies above the resonance, the oscillation can no longer catch up with the external field, and thus this mode no longer contributes to the cancelation of the electric field, which leads to a lower value in the real part of $\varepsilon(\omega)$. Therefore, the real part, $\varepsilon^{\prime}(\omega)$, steps down to a lower value with each resonance, whereas the imaginary part, $\varepsilon^{\prime \prime}(\omega)$, has successive absorption peaks.

For systems composed of polar molecules, the lowest frequency motion that leads to a dielectric effect is perhaps the reorientation. Therefore, the system starts with a (large) static dielectric constant in the real part, with Debye-like relaxation accounting for the rotational relaxation, followed perhaps by low and high-frequency vibrations that can contribute to the polarity, finally reaching the optical frequency such that it is no longer relevant to the ET problem.

The mathematical description for the above process, (i.e., a resonance in the imaginary part together with a step down in the real part of the response function) can be described by the KramerKronig relationship, which arises from the general causality of the response function, ${ }^{57,58}$ but will not be recapitulated here.

\subsection{Implication from dielectric dispersion}

The dielectric response for many commonly seen solvents has been studied extensively. The absorption spectra and dielectric constant at all the frequency ranges have been reported, offering an "experimental" version for the spectral density $J(\omega) .{ }^{51} J(\omega)$ can also be calculated from molecular dynamics (MD) simulation. ${ }^{59}$ Both offer useful information for charge-transfer behavior.

With experimental results, we include the spectral density function $J(\omega)$ for 5 different solvents as shown in Fig. 2. The experimental dielectric data were obtained from ref. 60-70. From Fig. 2 dielectric response spectra for the polar solvents have a large component for the low frequency region $\left(\leq 1000 \mathrm{~cm}^{-1}\right.$ for water, $\leq 2-300 \mathrm{~cm}^{-1}$ for methanol and acetonitrile). The lowfrequency response consists of intermolecular modes such as constrained translational and rotational modes. For polar molecules, an external field may induce an orientational motion, leading to a dielectric response. These are known to contribute significantly to the dynamic Stokes shift in time-dependent fluorescence. ${ }^{51,52}$

The intramolecular bending and $\mathrm{OH}$ stretching bands are also seen for both water and methanol, which are broader than the vibrational bands in the nonpolar system. All of these bands contribute to $\lambda_{\text {out }}$.

For nonpolar or weakly polar systems, sharp, narrow vibrational bands occur in the high-frequency area, and the low-frequency responses are very weak. For benezene and toluene, the largest contribution is from a band near $700 \mathrm{~cm}^{-1}$, which has been assigned as the out-of-plane $\mathrm{CH}$ bending. Indeed the $\mathrm{C}-\mathrm{H}$ bond is weakly polar, and its bending can create a polarization in response to a change in the solute. With these results, one might conclude that the high-frequency intramolecular vibration is important for the nonpolar solvation, whereas the influence is not so great for the low-frequency, orientational, or acoustic phonon-like region. However, the dielectric response model has its own assumption and limitations. For periodic or MD simulations, some response is still observed in the low-frequency region, as outlined below.

\section{Computational approaches and their findings}

The electron-phonon coupling can be evaluated nowadays using various computational approaches, for both Holstein and Peierls types of coupling. Here we summarize the available observations and limitations of these approaches in Table 1, with the detailed discussion as follows. 

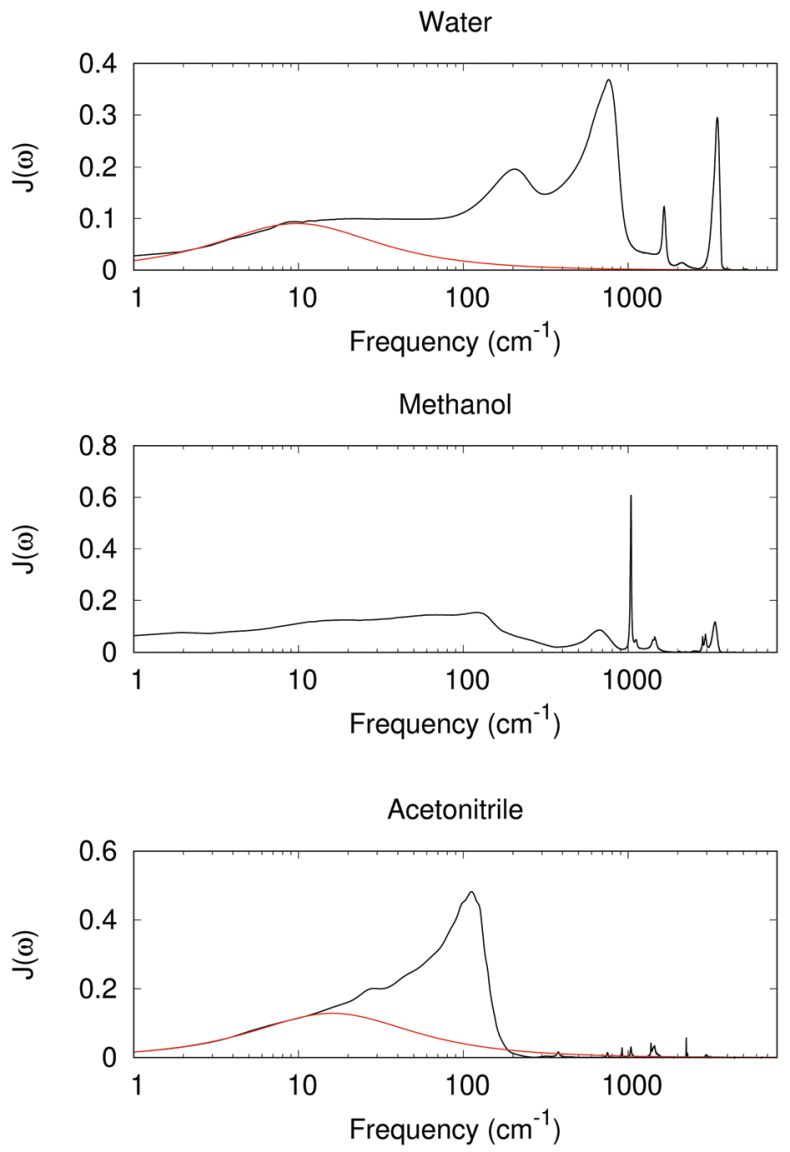

Toluene
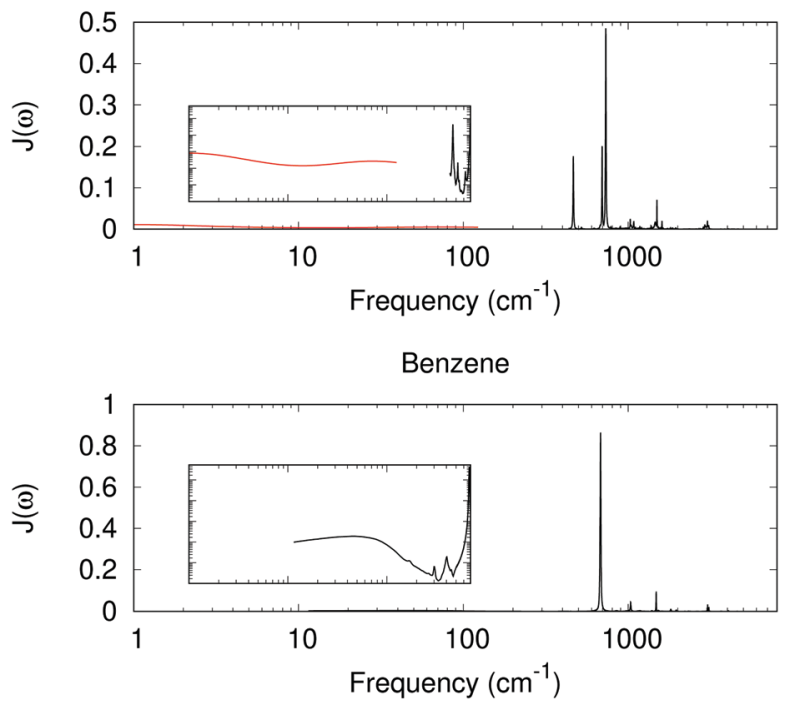

Fig. $2 \operatorname{Im}[1 / \varepsilon(\omega)]$ as a model for $J(\omega)$ with experimentally measured $\varepsilon(\omega)$ (black lines, data derived from ref. 52, 60, 63, 64, 66-68 and 70). At low frequency, a red curve for the Debye or multiple Debye model is included, with parameters from ref. 62 and 65 for water and acetonitrile, and interpolation and adjustment to $25^{\circ} \mathrm{C}$ according to ref. 69 . Inset: log-log scaled plots between 1 and $1000 \mathrm{~cm}^{-1}$ and $\operatorname{Im}[1 / \varepsilon(\omega)]$ between 0.0001 and 1 .

\subsection{Gas-phase calculations}

With the convenience of modern quantum chemistry computational programs, $\lambda_{\text {in }}$ can be routinely computed with geometry optimization in both reactant and product states, followed by a projection of the structural difference to the vibrational modes, as mentioned above in Section 2.1. Such results have been seen in many works. ${ }^{37,71,72}$

The commonly seen charge transporting materials often involve the aromatic $\pi$ moiety that has alternate $\mathrm{C}-\mathrm{C}$ single and double bonds. Ionization or excitation in these molecules generally involves a change in this alternacy: shorter single bonds and longer double bonds. Therefore, it is common to see a large contribution from this alternating $\mathrm{C}-\mathrm{C}$ stretch mode in the projection $\lambda_{\text {in }}$, leading to peaks in the region of 1300-1500 $\mathrm{cm}^{-1}$, whereas many other modes could have some contribution as well. ${ }^{73,74}$

For a mode with large vibration frequency, the population will mainly reside at the zero-point energy level, thus resulting in weak temperature dependence. Such a behavior was believed to contribute to the weak or even inverse temperature dependence reported in charge mobility in molecular crystals. ${ }^{72}$ However, such a model ignores the low-frequency modes which mainly arise from intermolecular libration or reorientation in response to a change in the charge position, an effect that exists predominantly in any condensed phase as further discussed below.

The electronic coupling calculation ${ }^{75}$ is becoming routinely available in many quantum chemistry packages, but the off-diagonal Peierls type of vibronic coupling has been also reported. ${ }^{73,74,76}$ In most of these works, the electronic coupling factor was scanned via a few degrees of freedom between two organic molecules. The scan is typically performed with planar $\pi$-conjugated organic molecules with one or two degrees of freedom in their relative position of $\pi-\pi$ stacking, and oscillatory coupling is observed owing to the nature of wavefunctions in the HOMO or LUMO, thus showing the importance of the intermolecular modes in a condensed array of molecules. Another well-known property is the exponential intermolecular distance dependence of the electronic coupling. ${ }^{77}$ These observed behaviors contribute to important Peierls coupling for the intermolecular acoustic-like modes. A direct computation for the nuclear derivative for the electronic coupling is available nowadays, ${ }^{78,79}$ which allows for direct computation for the Peierls coupling with any given vibrational mode, which would facilitate such characterization without having to scan for the nuclear degrees of freedom.

\subsection{Periodic boundary calculations}

In addition to amorphous solid states, charge mobilities have also been studied with single molecular crystals, ${ }^{3,80-83}$ which allows for better insights and understanding because the intermolecular structures are known. Thus, mobilities in crystals are excellent testbeds for theoretical predictions. Much theoretical and computational works have been developed for predicting properties for polarons for molecular crystals. ${ }^{34,84-88}$

Computational schemes for electron-phonon coupling for periodic systems have also been developed. ${ }^{89-91}$ In general, the electron-phonon coupling is a function of the band and the reciprocal vector for the electron and the band and the reciprocal vector for the phonon. A lot of such works have been reported for covalent systems such as inorganic semiconductors 
Table 1 Contribution of and limitations to various computational approaches for electron-phonon coupling

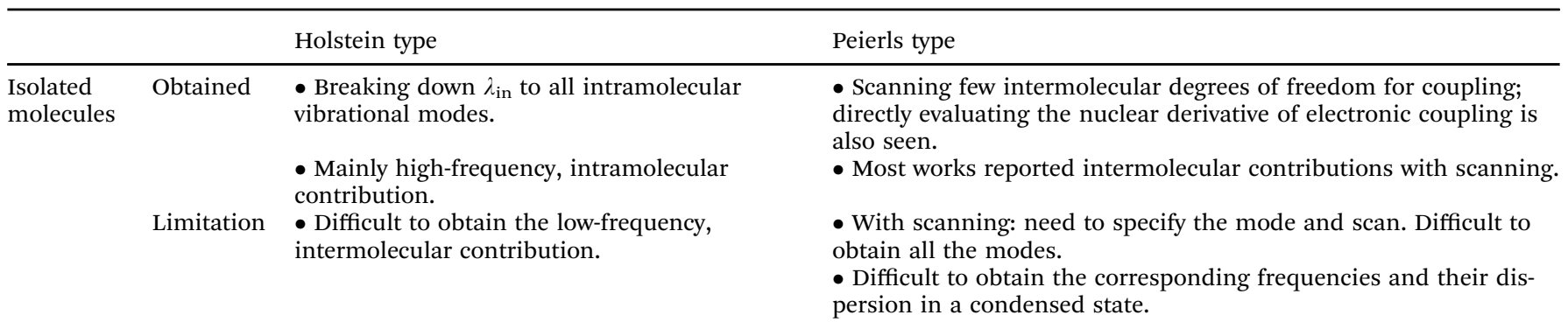

Periodic Obtained General approaches can account for models contributions from all phonons, including both optical (intramolecular) and acoustic (intermolecular) phonons

Limitation - Can be difficult to derive "chemical insights" in real space.

With MD Obtained Depending on the setting, can obtain purely simulation outer-sphere component, or both inter and intra-molecular contribution

Limitation $\bullet$ Quality depends on the force field used.
- Can fully account for all modes for the off-diagonal matrix elements.

- Involves 3 sets of indexes for the reciprocal vectors and corresponding electron and phonon bands. Even more complicated to gain chemical insights.

- The off-diagonal coupling can be obtained from MD snap shots. - Mainly arises from low-frequency, intermolecular motions

- Large cost of quantum chemistry calculation, and therefore, poor ensemble averaging quality. or graphene, ${ }^{92,93}$ whereby the rigid covalent bonds are likely to offer a steep electron phonon coupling. Such approaches can be used for organic molecular crystals, but a careful treatment of the density functionals for a good intermolecular dispersion interaction would be necessary.

A direct evaluation for the electron-phonon coupling in a periodic system can also be achieved by using derivative coupling ${ }^{94}$ or directly accounting for the matrix elements. ${ }^{95}$ Both diagonal and off diagonal electron-phonon couplings were reported in these works. The diagonal Holstein type of electron-phonon coupling is affected by both the low- and high-frequency modes, whereas the coupling for off-diagonal elements, the Peierls type of coupling, is mostly in the low-frequency acoustic phonons. ${ }^{94,95}$

\subsection{MD studies}

MD simulation has been a useful tool to offer structures and many quantities from the ensemble. In principle, function $J(\omega)$ can be obtained by the Fourier transformation of the nonequilibrium trajectory $\Delta E_{\text {int }}$, equivalent to $X$ defined in eqn (23), right after an electron is transferred to the acceptor. With the fluctuation-dissipation theorem, it is equivalently calculated by the autocorrelation function depicted in eqn (26). This approach has been commonly adopted and a good number of results have been reported. Such spectral density functions may be sensitive to the forcefield used, ${ }^{96}$ and thus the results should be taken cautiously. Such an approach has been important to elucidate the dynamics of charge-transfer systems. ${ }^{97,98}$

For example, the spectral densities have been reported for water and methanol..$^{59,99}$ In these early works, the general features are remarkably similar to those depicted in Fig. 2: an intramolecular contribution as well as low-frequency intermolecular bands. Nevertheless, such results imply that both inter- and intramolecular bands contribute to the dielectric response, or to the reorganizational energy for an ET process.

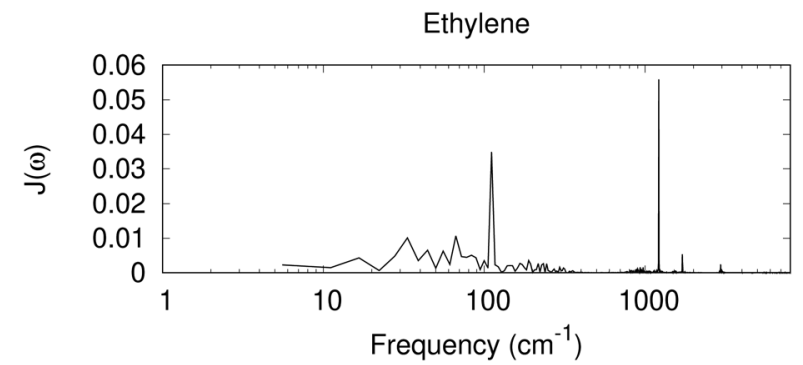

Fig. 3 Spectral density derived from a MD simulation for a periodic box of small nonpolar molecules of ethylene. Shown is the Fourier transformation of the autocorrelation function $C(t)$, scaled by $\operatorname{coth}(\beta \hbar \omega / 2)$ (eqn (26)), in arbitrary units.

In Fig. 3, we have included the spectral density derived from a Fourier transformation of the autocorrelation function $C(t)$, for ethylene, a nonpolar molecule in its liquid state, $\|$ as a test case for the nonpolar system. In this case, sharp high-frequency peaks are seen, similar to those with the dielectric response for benzene and toluene. The $1200 \mathrm{~cm}^{-1}$ peak for the $\mathrm{CH}_{2}$ rocking mode, together with weaker peaks near $1700 \mathrm{~cm}^{-1}$ for possibly the $\mathrm{C}=\mathrm{C}$ stretching, and $2900 \mathrm{~cm}^{-1}$ for $\mathrm{C}-\mathrm{H}$ stretching modes, is observed.

An interesting feature in Fig. 3 is the diffuse signal at the low-frequency region, at about $100 \mathrm{~cm}^{-1}$ and below. In contrast to the spectral density derived from the dielectric response for the nonpolar benzene that has nearly zero contribution in this region, with a similar nonpolar system, and similar to the direct

|| The simulation was performed using the Berendsen NPT ensemble, with temperature set at $130 \mathrm{~K}$ (for a liquid state) and pressure set at $1 \mathrm{bar}$, under optimized potentials for the liquid simulations force field ${ }^{100}$ using GROMACS (v 2016.4). ${ }^{101} \Delta E$ was simulated with the Coulomb interaction of a cationic ethylene (modeled by Mulliken population analysis via DFT at the LC-BLYP/DZ* level ${ }^{102,103}$ ) and the equilibrated surrounding molecules. 
calculation for electron-phonon coupling for nonpolar molecular crystals $^{94,95}$ a low-frequency response is seen with MD. The current dielectric response model assumes that the surrounding medium is a homogeneous dielectric, and the solute charge is assumed to be in a spherical cavity. In $\mathrm{MD}, \Delta E$ collected includes all the electrostatic interaction between a cationic ethylene and the surrounding neural molecules. Near the cation, a change in the orientation of a neutral ethylene leads to a different electrostatic interaction. It is also possible that higher order polarity effects could contribute (e.g. the quadruple moment could interact with a spatial change in the electric field). Therefore, low-frequency orientational or translational motion can contribute. Omitting such low-frequency contribution, even for nonpolar systems, might lead to an incorrect description for the system.

The off-diagonal electron-phonon coupling has also been studied with MD simulation and INDO/S semiempirical Hamiltonian calculations, or DFT. ${ }^{36,41,104}$ Again, the importance of lowfrequency modes close or under $100 \mathrm{~cm}^{-1}$ is observed, similar to those observed with periodic first-principle calculations. ${ }^{94,95}$ With the off-diagonal element of the Hamiltionian, the electronic coupling is sensitive to the relative position and orientation of the two molecules where ET is being considered, and thus, the low-frequency modes are important in this case. A future possibility is to use machine learning for a better ensemble averaging and higher resolution in the spectral density function. ${ }^{105}$

The quality of MD simulation results highly depends on the force field used. ${ }^{96}$ A polarizable force field should offer a better solution. Meanwhile, the development for combined quantum mechanical/molecular mechanical (QM/MM) calculations ${ }^{106}$ is worth noting. QM/MM allows for treating one or several molecules using a high-level method while accounting for the effects of the rest, which will offer a much better description for the reorganization $\lambda$ and its break-down, electron-phonon coupling factors $g$. By combining $\mathrm{QM} / \mathrm{MM}$ with $\mathrm{MD}$, a spectral density function can be obtained. This approach has been shown useful to simulate the spectra for pigment-binding proteins, and it can also be useful for describing ET systems. ${ }^{107}$

\section{Conclusions}

Here we summarize the consideration and description for reorganization energy, electron-phonon coupling, and spectral density for ET problems. The inner-shell contribution is from high-frequency intramolecular vibrational modes, and the outershell contribution is mainly low-frequency intermolecular modes. The traditional understanding of polar solvents includes an intense low-frequency Debye dielectric response and responses from high-frequency vibrational modes. However, for non-polar systems commonly seen in modern charge-transporting materials, a number of weak contributions from the vibration is predicted from the dielectric response model, and low-frequency contributions were reported via a direct account of electron-phonon coupling, or MD simulation. The low-frequency bands are also important for offdiagonal, Peierls couplings. As summarized in Fig. 4, these characteristics could facilitate future works in modeling and

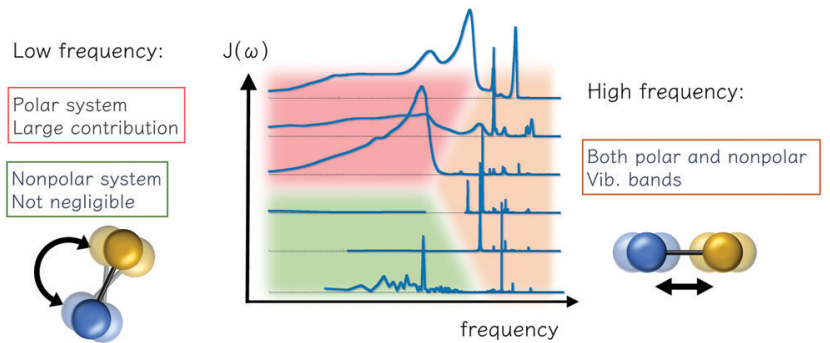

Fig. 4 Various contributions to the outer reorganization energy of an electron transfer system and their theoretical and computational aspects: the low-frequency reorientational and high-frequency vibrational contributions are observed. For polar environments both can be important, and for the nonpolar solvent, the low-frequency is important, depending on the model and computational settings, and such contribution could have bene overlooked.

simulating with realistic electron-phonon settings for good predictions in charge-transporting dynamics.

\section{Conflicts of interest}

There are no conflicts to declare.

\section{Acknowledgements}

The support from the Academia Sinica Investigator award (AS-IA-106-M01) and the Ministry of Science and Technology of Taiwan (project 105-2113-M-001-009-MY4) is gratefully acknowledged. Data for Fig. 3 were kindly provided by Chun-I Wang and Yi-Siang Wang.

\section{References}

1 R. A. Marcus, J. Chem. Phys., 1956, 24, 966-978.

2 R. A. Marcus and N. Sutin, Biochim. Biophys. Acta, 1985, 811, 265.

3 M. E. Gershenson, V. Podzorov and A. F. Morpurgo, Rev. Mod. Phys., 2006, 78, 973-989.

4 S. Sergeyev, W. Pisula and Y. H. Geerts, Chem. Soc. Rev., 2007, 36, 1902-1929.

5 J. D. Myers and J. Xue, Polym. Rev., 2012, 52, 1-37.

6 A. R. Murphy and J. M. J. Fréchet, Chem. Rev., 2007, 107, 1066.

7 S.-C. Lo and P. L. Burn, Chem. Rev., 2007, 107, 1097.

8 H. Spanggaard and F. C. Krebs, Sol. Energy Mater. Sol. Cells, 2004, 83, 125.

9 S. R. Forrest, Nature, 2004, 428, 911.

10 C. H. Teh, R. Daik, E. L. Lim, C. C. Yap, M. A. Ibrahim, N. A. Ludin, K. Sopian and M. A. M. Teridi, J. Mater. Chem. A, 2016, 4, 15788-15822.

11 S. Sze and K. N. Kwok, Physics of Semiconductor Devices, Wiley Online Library, 3rd edn, 2007.

12 L. Wang and D. Beljonne, J. Chem. Phys., 2013, 139, 064316.

13 G. Granucci and M. Persico, J. Chem. Phys., 2007, 126, 134114.

14 J. C. Tully, J. Chem. Phys., 2012, 137, 22 A301.

15 L. Wang, A. Akimov and O. V. Prezhdo, J. Phys. Chem. Lett., 2016, 7, 2100-2112. 
16 L. Wang, J. Qiu, X. Bai and J. Xu, Wiley Interdiscip. Rev.: Comput. Mol. Sci., 2020, 10, e1435.

17 H. Oberhofer, K. Reuter and J. Blumberger, Chem. Rev., 2017, 117, 10319-10357.

18 L. Wang, R. Long and O. V. Prezhdo, Annu. Rev. Phys. Chem., 2015, 66, 549-579.

19 J. Spencer, F. Gajdos and J. Blumberger, J. Chem. Phys., 2016, 145, 064102.

20 S. Giannini, A. Carof and J. Blumberger, J. Phys. Chem. Lett., 2018, 9, 3116-3123.

21 S. Giannini, A. Carof, M. Ellis, H. Yang, O. G. Ziogos, S. Ghosh and J. Blumberger, Nat. Commun., 2019, 10, 1-12.

22 A. A. Ovchinnikov and M. Y. Ovchinnikova, Sov. Phys. JEPT, 1969, 29, 688-693.

23 J. Ulstrup and J. Jortner, J. Chem. Phys., 1975, 63, 4358-4368.

24 J. N. Gehlen and D. Chandler, J. Chem. Phys., 1992, 97, 4958-4963.

25 Y. Georgievskii, C.-P. Hsu and R. A. Marcus, J. Chem. Phys., 1999, 110, 5307-5317.

26 M. Bixon and J. Jortner, Chem. Phys., 1993, 176, 467-481.

27 R. A. Kuharski, J. S. Bader, D. Chandler, M. Sprik, M. L. Klein and R. W. Impey, J. Chem. Phys., 1988, 89, 3248-3257.

28 R. A. Marcus, J. Chem. Phys., 1965, 43, 679-701.

29 R. A. Marcus, Faraday Discuss. Chem. Soc., 1982, 74, 7-15.

30 A. J. Leggett, S. Chakravarty, A. T. Dorsey, M. P. A. Fisher,

A. Garg and W. Zwerger, Rev. Mod. Phys., 1987, 59, 1-85.

31 T. Holstein, Ann. Phys., 1959, 8, 325-342.

32 T. Holstein, Ann. Phys., 1959, 8, 343-389.

33 Y. C. Cheng and R. J. Silbey, J. Chem. Phys., 2008, 128, 114713.

34 N. Prodanovicć and N. Vukmirovicć, Phys. Rev. B, 2019, 99, 104304.

35 E. Mozafari and S. Stafstroöm, Phys. Lett. A, 2012, 376, 1807-1811.

36 A. Troisi, Adv. Mater., 2007, 19, 2000-2004.

37 Z. Shuai, L. Wang and Q. Li, Adv. Mater., 2011, 23, 1145.

38 E. Mozafari and S. Stafstroöm, J. Chem. Phys., 2013, 138, 184104.

39 Y. Zhao, D. W. Brown and K. Lindenberg, J. Chem. Phys., 1994, 100, 2335-2345.

40 R. W. Munn and R. Silbey, J. Phys. Chem., 1985, 83, 1843-1853. 41 A. Troisi and G. Orlandi, Phys. Rev. Lett., 2006, 96, 086601. 42 Y. Yao, N. Zhou, J. Prior and Y. Zhao, Sci. Rep., 2015, 5, 1-10. 43 S. Fratini and S. Ciuchi, Phys. Rev. Lett., 2003, 91, 256403. 44 H. Ishii, N. Kobayashi and K. Hirose, Phys. Rev. B: Condens. Matter Mater. Phys., 2013, 88, 205208.

45 K. Hannewald and P. A. Bobbert, Phys. Rev. B: Condens. Matter Mater. Phys., 2004, 69, 075212.

46 S. Mukamel, Principles of Nonlinear Optical Spectroscopy, Oxford University Press, 1995.

47 S. Jang and Y.-C. Cheng, Wiley Interdiscip. Rev.: Comput. Mol. Sci., 2012, 3, 84-104.

48 T. Joo, Y. Jia, J.-Y. Yu, M. J. Lang and G. R. Fleming, J. Chem. Phys., 1996, 104, 6089-6108.

49 A. A. Stuchebrukhov and X. Song, J. Chem. Phys., 1994, 101, 9354-9365.

50 T. Ichiye, J. Chem. Phys., 1996, 104, 7561-7571.
51 C.-P. Hsu, X. Song and R. A. Marcus, J. Phys. Chem. B, 1997, 101, 2546-2551.

52 C.-P. Hsu, Y. Georgievskii and R. A. Marcus, J. Phys. Chem. A, 1998, 102, 2658-2666.

53 D. Chandler, Introduction to Modern Statistical Mechanics, Oxford University Press, 1987.

54 R. Kubo, Rep. Prog. Phys., 1966, 29, 255.

55 R. Kubo, M. Toda and N. Hashitsume, Statistical Physics II Nonequilibrium Statistical Mechanics, Springer-Verlag Berlin Heidelberg, 1991.

56 B. Bagchi, D. W. Oxtoby and G. R. Fleming, Chem. Phys, 1984, 86, 257-267.

57 J. S. Toll, Phys. Rev., 1956, 104, 1760-1770.

58 R. Kronig, J. Opt. Soc. Am., 1926, 12, 547-557.

59 K. Ando, J. Chem. Phys., 1997, 106, 116-126.

60 J. E. Bertie and Z. Lan, Appl. Spectrosc., 1996, 50, 1047-1057.

61 C. G. Malmberg and A. A. Maryott, J. Res. Natl. Bur. Stand., 1956, 56, 2641.

62 H. J. Liebe, G. A. Hufford and T. Manabe, Int. J. Infrared Millimeter Waves, 1991, 12, 659-675.

63 J. E. Bertie, S. L. Zhang, H. H. Eysel, S. Baluja and M. K. Ahmed, Appl. Spectrosc., 1993, 47, 1100-1114.

64 J. E. Bertie and Z. Lan, J. Phys. Chem. B, 1997, 101, 4111-4119.

65 J. Barthel, M. Kleebauer and R. Buchner, J. Solution Chem., 1995, 24, 1-17.

66 S. Ikawa, T. Ohba, S. Tanaka, Y. Morimoto, K. Fukushi and M. Kimura, Int. J. Infrared Millimeter Waves, 1985, 6, 287-306.

67 P. Firman, A. Marchetti, M. Xu, E. M. Eyring and S. Petrucci, J. Phys. Chem., 1991, 95, 7055-7061.

68 J. E. Bertie, Y. Apelblat and C. D. Keefe, J. Mol. Struct., 2005, 750, 78-93.

69 V. A. Santarelli, J. A. MacDonald and C. Pine, J. Chem. Phys., 1967, 46, 2367-2375.

70 J. E. Bertie and C. D. Keefe, J. Mol. Struct., 2004, 695-696, 39-57.

71 N. E. Gruhn, D. A. Da Silva Filho, T. G. Bill, M. Malagoli, V. Coropceanu, A. Kahn and J.-L. Breédas, J. Am. Chem. Soc., 2002, 124, 7918-7919.

72 G. Nan, X. Yang, L. Wang, Z. Shuai and Y. Zhao, Phys. Rev. B: Condens. Matter Mater. Phys., 2009, 79, 115203.

73 J. Cornil, D. Beljonne, J.-P. Calbert and J.-L. Breédas, Adv. Mater., 2001, 13, 1053-1067.

74 J. L. Brédas, J. P. Calbert, D. A. da Silva Filho and J. Cornil, Proc. Natl. Acad. Sci. U. S. A., 2002, 99, 5804.

75 C.-P. Hsu, Acc. Chem. Res., 2009, 42, 509-518.

76 B.-C. Lin, C.-P. Cheng, Z.-Q. You and C.-P. Hsu, Phys. Chem. Chem. Phys., 2011, 13, 20704-20713.

77 Z. Q. You, Y. H. Shao and C. P. Hsu, Chem. Phys. Lett., 2004, 390, 116-123.

78 A. F. Morrison and J. M. Herbert, J. Phys. Chem. Lett., 2017, 8, 1442-1448.

79 N. Bellonzi, E. Alguire, S. Fatehi, Y. Shao and J. E. Subotnik, J. Chem. Phys., 2020, 152, 044112.

80 V. C. Sundar, J. Zaumseil, V. Podzorov, E. Menard, R. L. Willett, T. Someya, M. E. Gershenson and J. A. Rogers, Science, 2004, 303, 1644. 
81 V. Podzorov, E. Menard, A. Borissov, V. Kiryukhin, J. A. Rogers and M. E. Gershenson, Phys. Rev. Lett., 2004, 93, 086602.

82 J. Y. Lee, S. Roth and Y. W. Park, Appl. Phys. Lett., 2006, 88, 252106.

83 J. Takeya, M. Yamagishi, Y. Tominari, R. Hirahara, Y. Nakazawa, T. Nishikawa, T. Kawase, T. Shimoda and S. Ogawa, Appl. Phys. Lett., 2007, 90, 102120.

84 V. M. Kenkre, J. D. Andersen, D. H. Dunlap and C. B. Duke, Phys. Rev. Lett., 1989, 62, 1165-1168.

85 F. Ortmann, F. Bechstedt and K. Hannewald, Phys. Rev. B: Condens. Matter Mater. Phys., 2009, 79, 235206.

86 F. Ortmann, F. Bechstedt and K. Hannewald, New J. Phys., 2010, 12, 023011.

87 Y.-C. Wang and Y. Zhao, Phys. Rev. B, 2020, 101, 075205.

88 Y.-J. Zhong, C.-F. Lan, B.-C. Lin, C.-D. Hu, Y.-C. Cheng and C.-P. Hsu, Adv. Quantum Chem., 2020, 81, 219-241.

89 F. Giustino, M. L. Cohen and S. G. Louie, Phys. Rev. B: Condens. Matter Mater. Phys., 2007, 76, 165108.

90 F. Giustino, Rev. Mod. Phys., 2017, 89, 015003.

91 J. Noffsinger, F. Giustino, B. D. Malone, C.-H. Park, S. G. Louie and M. L. Cohen, Comput. Phys. Commun., 2010, 181, 2140-2148.

92 T. Gunst, T. Markussen, K. Stokbro and M. Brandbyge, Phys. Rev. B, 2016, 93, 035414.

93 C.-H. Park, N. Bonini, T. Sohier, G. Samsonidze, B. Kozinsky, M. Calandra, F. Mauri and N. Marzari, Nano Lett., 2014, 2014, 3.
94 A. F. Morrison and J. M. Herbert, J. Chem. Phys., 2017, 146, 224110.

95 P. Ordejoón, D. Boskovic, M. Panhans and F. Ortmann, Phys. Rev. B, 2017, 96, 035202.

96 P. Zarzycki and B. Gilbert, Phys. Chem. Chem. Phys., 2020, 22, 1011-1018.

97 J. N. Gehlen, M. Marchi and D. Chandler, Science, 1994, 263, 499-502.

98 J. S. Bader and D. Chandler, Chem. Phys. Lett., 1989, 157, 501-504.

99 K. Ando, J. Chem. Phys., 2001, 114, 9040-9047.

100 W. L. Jorgensen, D. S. Maxwell and J. Tirado-Rives, J. Am. Chem. Soc., 1996, 118, 11225-11236.

101 M. J. Abraham, T. Murtola, R. Schulz, S. Paáll, J. C. Smith, B. Hess and E. Lindahl, SoftwareX, 2015, 1-2, 19-25.

102 A. Savin, in Recent Developments and Applications of Modern Density Functional Theory, ed. J. Seminario, Elsevier Science, Amsterdam, 1996, pp. 327-358.

103 H. Iikura, T. Tsuneda, T. Yanai and K. Hirao, J. Chem. Phys., 2001, 115, 3540-3544.

104 A. Troisi and G. Orlandi, J. Phys. Chem. A, 2006, 110, 4065-4070.

105 C.-I. Wang, M. K. E. Braza, G. C. Claudio, R. B. Nellas and C.-P. Hsu, J. Phys. Chem. A, 2019, 123, 7792-7802.

106 H. Lin and D. G. Truhlar, Theor. Chem. Acc., 2006, 117, 185-199.

107 A. M. Rosnik and C. Curutchet, J. Chem. Theory Comput., 2015, 11, 5826-5837. 